\title{
Evaluación del diseño e implementación de la metodología flipped-classroom en la formación del profesorado de ciencias sociales
}

\section{Assessment of the design and implementation of the flipped- classroom methodology in the training of teachers of social sciences}

\author{
José Monteagudo Fernández \\ Universidad de Murcia. Murcia, España \\ jose.monteagudo@um.es \\ Cosme Jesús Gómez Carrasco \\ Universidad de Murcia. Murcia, España \\ cjgomez@um.es \\ Pedro Miralles Martínez \\ Universidad de Murcia. Murcia, España \\ pedromir@um.es
}

\begin{abstract}
Resumen
El presente trabajo aborda una experiencia de aula sobre la evaluación del diseño e implementación de varias flipped-classroom como método didáctico con alumnado de educación superior. Como instrumento de recogida de datos se utilizó un cuestionario que se administró a tres de los grupos de la asignatura "Metodología didáctica para la enseñanza de las ciencias sociales" de 3. ${ }^{\circ}$ curso del Grado en Educación Primaria de la Universidad de Murcia. Los resultados indican que, a pesar de la escasa participación del alumnado, relatada por ellos mismos, los estudiantes valoran muy positivamente las sesiones de flipped-classroom desarrolladas, así como el propio método para conseguir aprendizajes más significativos. De esta forma concluimos que es necesario seguir implementando este método de enseñanza con los futuros docentes para conseguir así un profesorado mejor formado que lleve a las aulas de primaria estrategias activas de aprendizaje.

Palabras clave

Flipped-classroom, formación del profesorado, didáctica de las ciencias sociales, enfoque basado en el estudiante.
\end{abstract}

\begin{abstract}
This paper is about a classroom experience on the assessment of the design and implementation of several flipped-classrooms as didactic method with students of higher education. We used a questionnaire as instrument for the data collection, which was administered to three of the groups of the subject "Metodología didáctica para la enseñanza de las ciencias sociales" of $3^{\text {rd }}$ year of the Degree in Primary Education of the University of Murcia. The results indicate that, in spite of the low participation of students, related by themselves, the students value very positively the sessions of flipped-classroom developed, as well as the own method to obtain more significant learning. In this way, we conclude that it is necessary to continue to implement this teaching method with the future teachers to obtain a better-trained teaching staff that will take to the primary education classrooms active learning strategies.
\end{abstract}

Key words

Flipped-classroom, Teacher training, Social Sciences Education, Student Approaches to Learning. 


\section{Introducción}

Actualmente las tecnologías ofrecen enormes posibilidades educativas y los estudiantes presentan un alto grado de dominio de las mismas, ya que viven en una combinación de espacios digitales y reales. ¿Por qué no aprovecharlo para las tareas docentes? En este sentido la flipped-classroom es un modelo pedagógico que transfiere el trabajo de determinados procesos de aprendizaje fuera del aula y utiliza el tiempo de clase, junto con la experiencia del docente, para facilitar y potenciar otros procesos de adquisición y práctica de conocimientos dentro del aula.

Pero darle la vuelta a una clase es mucho más que editar vídeos. De hecho, el componente más valioso es el mejor uso del tiempo de clase para que los estudiantes participen en actividades que impliquen el desarrollo de estrategias de aprendizaje de nivel superior. Nos encontramos así con un enfoque integral que combina la instrucción directa con métodos constructivistas, propiciando el aumento de la motivación e implicación del alumnado con los contenidos de las asignaturas.

Además de la mejora de la comprensión conceptual, la clase invertida facilita la aplicación de procedimientos y la puesta en marcha de otras habilidades cognitivas superiores mediante el trabajo cooperativo en el aula, también en educación superior, donde el empleo de estudios de caso, el aprendizaje basado en problemas y otros métodos y técnicas activos de aprendizaje permiten al alumnado, además de adquirir competencias cognoscitivas, un compromiso ético y motivación hacia el trabajo grupal (Sánchez, 2010). En la enseñanza centrada en el estudiante destaca el puzle de Aronson como técnica que potencia el protagonismo del alumno en su propio proceso educativo, pues sólo el estudiante alcanza sus objetivos si el resto de compañeros de grupo alcanzan también los suyos (Sánchez, 2010).

Desde la disciplina de las ciencias sociales estos métodos de indagación facilitan la formación de un pensamiento social asentado sobre los pilares de la causalidad, la intencionalidad, el relativismo y el pensamiento crítico y creativo (Santisteban, 2011); para de esta manera ayudar al alumnado a comprender los fenómenos sociales y aprender a vivir en sociedad, formando futuros ciudadanos y actores protagonistas críticos con la realidad que les ha tocado vivir.

Ello fue, precisamente, lo que se puso en práctica con el alumnado de Grado de Educación Primaria en la asignatura "Metodología didáctica para la enseñanza de las ciencias sociales", en la que se realizaron cuatro sesiones de flipped-classroom para desarrollar determinados aspectos de la materia de una forma que permitiera una mayor participación de los discentes y un mayor grado de profundidad en la asimilación de los contenidos. Experimentar este método didáctico en primera persona puede ayudar a que los docentes en formación, futuros maestros, lleven a las aulas de primaria estrategias que promuevan unos aprendizajes más significativos, que en las ciencias sociales se traducen en dotar al alumnado de perspectiva y elementos de juicio suficientes para tomar decisiones autónomas que pueden cambiar su entorno.

Evaluación del diseño e implementación de la metodología flipped-classroom en la formación del profesorado de ciencias sociales. José Monteagudo Fernández, Cosme Jesús Gómez Carrasco y Pedro Miralles Martínez.

Página 2 de 26 


\section{Marco teórico}

\subsection{Los enfoques de enseñanza-aprendizaje en Educación Superior}

Los enfoques de enseñanza y aprendizaje en Educación Superior están condicionados al menos por tres importantes factores. En primer lugar, por los cambios que en la última década se han producido a nivel social y tecnológico en lo que se denomina actualmente como sociedad de la información y la comunicación. En segundo lugar por el Espacio Europeo de Educación Superior (EEES), que establece un nuevo marco institucional y educativo basado en créditos ECTS y en la adquisición de competencias. En tercer lugar por los cambios pedagógicos asociados a estos dos primeros factores. Estos cambios pedagógicos exigen del profesorado universitario el manejo de herramientas y recursos adecuados a las nuevas transformaciones sociales y culturales. Como indican Moron, De Caro y Lagomarsino (2013), las buenas prácticas educativas con TIC son acciones complejas y multidimensionales, que requieren la planificación o programación como guía indispensable para llevar adelante la tarea de preparar las clases (Magadán, 2012), tomando decisiones de tipo curricular, pedagógicas y tecnológicas, en este orden según Mishra \& Koehler (2006). Pero también exigen del docente un nuevo enfoque de enseñanza centrada en el estudiante (Student Approaches to Learning, SAL).

El aprendizaje centrado en el alumnado proporciona oportunidades de aprendizaje que se ajustan a las necesidades e intereses de cada estudiante. Mediante este enfoque los alumnos aprenden de forma activa y los docentes se encargan de facilitar su aprendizaje. Es un cambio fundamental de enfoque en el que el alumnado es el principal protagonista del proceso de enseñanza-aprendizaje, mientras que el profesor asume su papel de guía, facilitador y orientador del mismo. Como indica Yáñiz (2008), el contexto institucional de la EEES y el enfoque basado en competencias facilita este paradigma (Rué, 2008; Perrenoud, 2008). Un paradigma que supone un cambio de perspectiva sobre el alumno y verlo como sujeto activo constructor de su proceso de aprendizaje. Además es importante diseñar contextos educativos que estimulen una implicación integral en el desarrollo de competencias (por lo tanto en conocimientos, habilidades y actitudes) del alumno en la construcción de dicho proceso (Yáñiz, 2008). Por lo tanto resulta más necesario que nunca conocer bien a esos alumnos de cara a crear contextos educativos (metodologías, sistemas de acompañamiento, relaciones y ambiente positivo de aprendizaje) que repercutan en la calidad de su proceso de aprendizaje universitario (García 2016).

Las concepciones iniciales que presentan el profesorado y el alumnado universitarios sobre qué es enseñar y qué es aprender influyen en las decisiones que, cotidianamente, se adoptan en uno u otro sentido. Por tanto, se utilizan unos enfoques de enseñanza y de aprendizaje que en un contexto educativo determinado se relacionan, y determinan la calidad y los resultados del proceso de enseñanza-aprendizaje. Es importante subrayar que el enfoque de aprendizaje no se constituye como una característica que el individuo posea, si no que se considera fruto de la interacción entre el individuo y su contexto de aprendizaje. Por tanto no es una característica estática o inamovible del sujeto. El desarrollo de un determinado enfoque de aprendizaje se relaciona con las experiencias educativas vividas por el alumno y posee, por lo tanto, una naturaleza contextual.

Evaluación del diseño e implementación de la metodología flipped-classroom en la formación del profesorado de ciencias sociales. José Monteagudo Fernández, Cosme Jesús Gómez Carrasco y Pedro Miralles Martínez.

Página 3 de 26 
También conviene señalar que la convivencia de ambos enfoques en una misma persona es posible ya que no son mutuamente excluyentes y dependen de las diferentes experiencias educativas alrededor del sujeto (Entwistle \& McCune, 2004; Biggs, 2005; García, 2016).

Para Gargallo, Garfella y Pérez (2006), hay tres cuestiones fundamentales que plantearse a la hora de abordar el problema de los procesos de aprendizaje: ¿cómo estudian y aprenden los estudiantes?, ¿qué modos de abordar el aprendizaje son más eficaces? y ¿por qué los estudiantes aprenden como lo hacen? Hernández Pina (2002) indica que las dos concepciones de enseñanza más aceptadas y practicadas por el profesorado universitario han sido la concepción cuantitativa y cualitativa. La concepción cuantitativa se relaciona con el profesor experto en su materia, que sabe comunicar, de forma fluida, los conocimientos asociados a su disciplina. Concibe la enseñanza como la simple transmisión de información y el proceso de aprendizaje se reduce a una mera acumulación de hechos y habilidades, ordenados de tal forma que estén disponibles en el momento oportuno. La concepción cualitativa de la enseñanza se vincula al profesor que no sólo transmite contenidos sino que se convierte en facilitador, que media y ayuda al estudiante a construir activamente significados para una mejor comprensión del mundo que le rodea, interpretando los contenidos, dándoles un significado y ayudándole en su proceso de interpretación de la realidad. Se trata de un docente que utiliza métodos para que el estudiante se implique activamente en esta evolución personal, favoreciendo la interacción con sus iguales y desarrollando actitudes y valores.

Además del polo centrado en el profesorado, también para Hernández Pina (2002) existe un polo centrado en el estudiante, en sus necesidades, orientado al aprendizaje, a su facilitación, dirigido al cambio conceptual y al desarrollo intelectual como aprendices independientes. Es lo que Entwistle (2000, págs. 343-344) denomina "concepción sofisticada", porque apoya un cambio conceptual más allá de la comprensión. Se trata de un acto creativo y global por medio del cual se llega a la experiencia personal del estudiante incluyendo aspectos afectivos y cognitivos.

Al igual que en las concepciones de enseñanza, las concepciones de aprendizaje de los estudiantes se incluyen en un continuo que abarca desde el aprendizaje como acumulación de información, que ha de ser memorizada y luego reproducida en el momento de la evaluación, hasta el aprendizaje como desarrollo personal, abstrayendo significados a través de un proceso interpretativo dirigido a la comprensión. Como indican González, Del Rincón y Del Rincón (2011), y manteniendo la estructura de estos dos enfoques tal y como señalan autores como Biggs, Kember \& Leung (2001), las investigaciones realizadas parecen indicarnos la existencia de una infinidad de perfiles de aprendizaje comprendidos en un continuo bipolar (Kember, 2000; Hernández Pina, 1999; Hernández Pina et al., 2002; Hernández Pina et al., 2006). Así, el alumnado, dando preferencia a motivaciones y estrategias de ambos enfoques, puede situarse más cerca de uno de ellos.

Romero et al. (2013) indica que en el contexto universitario los enfoques denominados como superficiales (surface), supondrían estudiar lo mínimo necesario para ser

Evaluación del diseño e implementación de la metodología flipped-classroom en la formación del profesorado de ciencias sociales. José Monteagudo Fernández, Cosme Jesús Gómez Carrasco y Pedro Miralles Martínez.

Página 4 de 26 
meramente capaz de reconocer y reproducir los materiales con objeto de superar la evaluación académica. Por el contrario, los enfoques de aprendizaje denominados como profundos (deep) consistirían en estudiar comprendiendo el significado del material que se quiere aprender y por tanto, buscando relaciones entre las ideas, reflexionando sobre los conceptos obtenidos en las clases, cuestionando lo que se lee, examinando los detalles para apoyar los argumentos que se hacen, etc.

El verdadero impacto de la corriente del SAL propuesto por el EEES es la posibilidad de relación que existe entre el enfoque mostrado y el resultado de aprendizaje obtenido tras la tarea. Es decir, parece ser que aquellos alumnos que optan por un enfoque profundo obtienen mejores resultados que aquellos que optan por un enfoque superficial, tal y como puede comprobarse en multitud de investigaciones (Biggs et al., 2001; Gargallo, Garfella y Pérez, 2006).

Como indica Guzmán y García (2017), la armonización de los sistemas universitarios en el EEES se enfrenta en el horizonte posterior a 2010 a nuevos y antiguos retos a los que ha de dar respuesta en cada uno de los países de la Unión Europea. Las transformaciones experimentadas en el contexto social y educativo con el cambio de siglo han obligado a las universidades a adoptar numerosos ajustes relacionados con la programación, la metodología y la evaluación en las distintas disciplinas. Dichos cambios en el EEES fueron motivados por la necesidad de incorporar al alumnado a una comunidad de práctica universitaria, que comparte actividades de lectura y escritura en un ámbito disciplinar, utilizando géneros y prácticas discursivos con sus respectivos códigos y convenciones (Curry \& Lillis, 2005).

Cuando hacemos referencia al alumnado universitario estamos hablando de educación de personas adultas-jóvenes y hemos de pensar en ellas como sujetos con creencias, teorías, experiencias y conocimientos previos, lo que impone la necesidad de proponer un tipo de enseñanza concebida más como facilitación y construcción de nuevos modos de pensamiento y acción que como transmisión para una asimilación acrítica por parte del alumno. Además, se han de proponer unas acciones que sirvan para el replanteamiento, revisión y reconstrucción de las creencias y conocimientos previos, etc. De ahí la necesidad de ofrecer un entorno de apoyo estimulante cuyo foco primario se centre en la indagación reflexiva como medio de desarrollo epistemológico y cognitivo. Por tanto, de esta situación deviene esencial diseñar una enseñanza concebida más como facilitación y construcción de nuevos modos de pensamiento y acción, en la línea de enseñar a pensar, que como transmisión de contenidos que debe asimilar el alumnado.

Para que se produzca un aprendizaje significativo y una asimilación efectiva de contenidos y competencias, el sujeto obtiene e intercambia información en un marco social, en relación con los demás. Vigotsky lo ha llamado aprendizaje coral (Zabalza, 2002). El estudiante universitario interacciona por medio del intercambio directo y presencial con el profesor y sus compañeros y también a través de soportes como libros, documentos, medios audiovisuales, Internet, etc. En definitiva, se van construyendo nuestras propias ideas a partir del contraste con los demás.

Evaluación del diseño e implementación de la metodología flipped-classroom en la formación del profesorado de ciencias sociales. José Monteagudo Fernández, Cosme Jesús Gómez Carrasco y Pedro Miralles Martínez.

Página 5 de 26 
Es complejo sistematizar en categorías cerradas los estilos de aprendizaje del alumnado universitario debido a su diversidad y complejidad. En opinión de Bolívar y Rojas (2008) los estudiantes universitarios tienen una determinada actitud y comportamiento sobre el entorno académico que los rodea. Cada uno de ellos posee características particulares, fisiológicas y psicológicas, que distinguen a unos de otros. Esto explica, en parte, las diferencias del cómo, cuándo y por qué aprenden. Es decir, podemos entender que los enfoques de aprendizaje son bastante sensibles al entorno y, por tanto, difíciles de entender sin una correcta contextualización (González, Del Rincón y Del Rincón, 2011). Cada estudiante responde a un estilo particular y predominante de aprendizaje que le permite interactuar con el escenario educativo (Juárez, Rodríguez, Escoto y Luna, 2017). Fuentes (2003) afirma que si las estrategias de aprendizaje son un plan de acción, flexible y con miras a alcanzar una meta, es pertinente pensar que pueden estar orientadas por el funcionamiento cognitivo del sujeto, por su manera de aproximarse a los problemas, por su modo peculiar de percibir y procesar la información, es decir por su estilo de aprendizaje (Juárez, Rodríguez, Escoto y Luna, 2017).

Como indican González, Del Rincón y Del Rincón (2011), parece lógico pensar que aquel profesor que pretenda mejorar su enseñanza en el marco teórico SAL debe pensar en qué debe hacer para que sus alumnos aprendan mejor. Ello supone poner el foco en el alumnado, su contexto y sus estrategias de asimilación de conocimientos, propiciando así una nueva concepción del proceso de enseñanza-aprendizaje en la que el eje fundamental de la reflexión docente va girando progresivamente de la enseñanza al aprendizaje (Ojeda et al., 2010).

Antes de iniciar un proceso de redefinición metodológica de una asignatura o materia es fundamental conocer y considerar las peculiaridades o características cognitivas concretas de los estudiantes, tales como sus enfoques de aprendizaje, su creatividad, su motivación o sus estilos de pensamiento, ya que éstas pueden determinar la eficacia de la metodología didáctica empleada (Albalate, Fageda, y Perdiguero, 2011; Demirbas \& Demirkan, 2007; García, Arias, Rodríguez, Fidalgo y Robledo, 2017).

\subsection{Las flipped-classroom en el contexto metodológico y de implementación de las TIC en Educación Superior}

Como ya se ha apuntado, el proceso de reconceptualización organizativa, estructural y funcional que impulsó hace ya casi dos décadas la Declaración de Bolonia (1999) parece inducir al uso en las aulas universitarias de métodos de enseñanza-aprendizaje de tipo activo ya que éstos, teóricamente, pretenden convertir al alumnado en el agente protagonista de su proceso de aprendizaje, dotándole de una mayor autonomía y poder de decisión y actuación en el mismo (García, Arias, Rodríguez, Fidalgo y Robledo, 2017).

Relacionado con ello, la formación del profesorado en el Grado de Educación Primaria debe demandar de los alumnos (los futuros docentes) nuevas formas de entender los procesos de enseñanza-aprendizaje, donde existan unos objetivos más globales e interdisciplinares (Ruiz, Rubia, Anguita y Fernández, 2010). Pero además en la formación del profesorado debe primar un equilibrio entre conocimientos, habilidades y destrezas. Para conseguir esta cuestión es necesario combinar estrategias que fomenten

Evaluación del diseño e implementación de la metodología flipped-classroom en la formación del profesorado de ciencias sociales. José Monteagudo Fernández, Cosme Jesús Gómez Carrasco y Pedro Miralles Martínez.

Página 6 de 26 
el conocimiento científico en el proceso de enseñanza, así como el dominio de un conjunto de métodos, técnicas y procedimientos para afrontar situaciones personales y profesionales (Zahonero y Martín, 2012).

En esta dirección ya incidieron Margalef y Pareja (2008), partiendo del análisis crítico de experiencias de innovación docente, cuando subrayaron que el compromiso por comprender, transformar y mejorar la práctica docente, a través de la indagación y reflexión colectiva, es un factor clave en los procesos de cambio educativo y en el desarrollo de metodologías activas de aprendizaje.

$\mathrm{Y}$ en este sentido, en una asignatura como en la que se ha implementado esta experiencia, "Metodología didáctica para la enseñanza de las ciencias sociales", en la que, grosso modo, se le muestra al alumnado cómo enseñar ciencias sociales en Educación Primaria, el principal objetivo debe ser el de trabajar con metodologías activas de aprendizaje para que los discentes aprendan habilidades y competencias en la enseñanza de ciencias sociales en Primaria desde una perspectiva constructivista. Pero además debe orientarse hacia la consecución de valores en el trabajo en equipo, y en la capacidad de comunicación y de reflexión sobre la enseñanza, sus métodos y planificación (León y Crisol, 2011). Si, como indica López (2007), el docente universitario debe protagonizar un giro pedagógico hacia la búsqueda de nuevas estrategias para desarrollar la creatividad, la calidad, las competencias y la colaboración, en las Facultades de Educación esta necesidad es mayor debido a la formación de futuros profesores.

Todo ello, unido a los cambios que se han producido a nivel social, ha conducido a una reflexión sobre la metodología didáctica que el docente debe llevar a cabo en el aula, pues no cabe duda de que los métodos de enseñanza deben adecuarse a las transformaciones sociales y culturales. De esta manera, el docente debe orientar y guiar al alumnado en su proceso de aprendizaje, debe enseñar -además de conocimientos y destrezas- herramientas y habilidades para que el alumnado sea autónomo, así como incorporar medios y recursos que faciliten la actividad docente en el aula. Esto exige que en la formación de estos profesionales se introduzcan competencias para diseñar experiencias de aprendizaje significativas que produzcan una comprensión duradera de estos procesos y así poder enfrentarse a las tareas diarias que se encontrarán en el aula (Del Moral, 2012).

Así las cosas, no es de extrañar que una de las principales competencias docentes que ha de adquirir el profesorado para desenvolverse correctamente en el aula (Cano, 2005; Fielden, 2001; Perrenoud, 2004; Torra et al., 2012) sea la competencia metodológica. Sin embargo esta competencia puede quedar vacía si el profesor no la une con el conocimiento de la materia que quiere enseñar. Ése es uno de los principales campos de actuación de las didácticas específicas o aplicadas, el de mostrar las estrategias metodológicas que permitan a los futuros docentes enseñar los contenidos esenciales de cada una de las materias desde distintos enfoques.

En el caso de las ciencias sociales la tradición escolar y académica heredada del siglo XIX ha insistido en que el aprendizaje de estas disciplinas se desarrolle desde una

Evaluación del diseño e implementación de la metodología flipped-classroom en la formación del profesorado de ciencias sociales. José Monteagudo Fernández, Cosme Jesús Gómez Carrasco y Pedro Miralles Martínez.

Página 7 de 26 
perspectiva principalmente teórica. De hecho, el enfoque práctico, así como las actividades a través del laboratorio/taller se ha reservado a las materias denominadas científicas y experimentales (Santacana, 2005). La articulación de los saberes escolares que debían enseñarse en las materias de geografía e historia se concibieron principalmente con la función de legitimar los estados-nación que fueron surgiendo desde finales del siglo XIX (Pérez Garzón, 2008; Boyd, 2000). Frente a estas disciplinas (geografía e historia) cuya enseñanza y aprendizaje se basaba casi exclusivamente en el relato, lectura y memoria, se situaban otras (ciencias y técnicas) que requerían de la experimentación y de las prácticas. El resultado fue que tan sólo las materias científicas se consideraban susceptibles de disponer de laboratorios y que recibieran esa caracterización de "experimentales" (Santancana, 2005). No obstante la enseñanza de las ciencias sociales debe dar un giro para mostrar su contenido como un proceso en construcción. Por ello Prats y Santacana (2011) insisten en que este conocimiento debe realizarse a través de la indagación, de métodos de análisis social y mostrando estos saberes más allá de la simple erudición y memorización de fechas, datos, nombres propios y definiciones conceptuales.

La incorporación de las TIC en todos los niveles del sistema educativo ha conformado una nueva cultura tecnológica que ha generado importantes esfuerzos en cuanto a adaptación a estos nuevos medios (Rodríguez-Correa y González-Sanmamed, 2013). De esta forma, se ha creado una conciencia generalizada acerca de la necesidad de formación para el uso de estas herramientas tanto por parte del profesorado como del alumnado, para lograr integrarlas de manera significativa a los procesos de enseñanza aprendizaje (Cabero y Marín, 2014). En caso contrario, dar la espalda a estos cambios tecnológicos, puede traer como consecuencia una desconexión de la dinámica de aprendizaje del alumnado. Como indican Artal, Casanova, Serrano y Romero (2017), apostar por metodologías activas de aprendizaje (Flipped-Classroom, Aprendizaje Basado en Problemas; Método de Proyectos; Estudios de Caso, etc.), y usar determinadas herramientas y aplicaciones TIC puede ayudar a solventar este problema.

La principal función de este tipo de formación, basada en el uso de las TIC, sería desarrollar en el docente y el alumno/a un sentido crítico en el uso y aprendizaje de los medios tecnológicos. Por tanto, existe la necesidad de vincular la teoría con la práctica, el feedback continuo y el perfeccionamiento día a día de las actividades que se desarrollarán en este tipo de propuestas formativas.

Por ello, las universidades españolas están poniendo en práctica innumerables acciones de innovación educativa, con el fin de superar los esquemas didácticos tradicionales y lograr una enseñanza de calidad, objetivo fundamental del proceso de convergencia europea que se está viviendo en la actualidad (Álvarez, Cuéllar, López, Adrada, Anguiano, Bueno, Comas, y Gómez, 2011).

Frecuentemente nos encontramos con algunos profesores que muestran resistencia al cambio e incluso a la idea de incorporar algunas TIC y plataformas de teleformación a sus prácticas. Según Selwyn, Marriot \& Marriot (2002) algunas de estas resistencias tendrán que ver en algún grado con las carencias de culturas institucionales en cuanto a la utilización de las TIC. Sin embargo otros estudios señalan por el contrario, que los

Evaluación del diseño e implementación de la metodología flipped-classroom en la formación del profesorado de ciencias sociales. José Monteagudo Fernández, Cosme Jesús Gómez Carrasco y Pedro Miralles Martínez.

Página 8 de 26 
profesores universitarios tienen actitudes positivas para la utilización e incorporación de las TIC en los procesos de formación (Jimoyiannis \& Komis, 2007; Banas, 2010).

Para disminuir estas resistencias, el docente deberá recibir una capacitación conceptual respecto a cómo las TIC y las plataformas de teleformación se comportan en los ambientes educativos, la cual ayudará a transformar las creencias que tengan respecto a estos medios, y le permitirá tomar decisiones para adaptar y buscar nuevas formas de utilización, y no ser meramente un reproductor de prácticas de enseñanzas elaboradas por otros (Cabero, 2014). Esta formación del profesorado deberá contemplar por lo tanto, un enfoque más global e integrador en el que se tenga en cuenta las dimensiones instrumental, pragmática, didáctica, metodológica, psicológica, actitudinal, investigadora y curricular (Del Moral y Villalustre, 2010); de esta forma, "se garantizará no solo la adquisición de competencias relacionadas con el uso y el manejo de herramientas tecnológicas (destrezas, habilidades, dominio de softwares, etc.), sino competencias personales vinculadas al proceso de aprendizaje del alumno y de enseñanza del profesorado" (Muñoz-Repiso, Gómez-Pablos, González, Martín, Rodero, Martín y Marcos, 2015, p. 86).

No cabe duda de que las dos principales funciones de internet -transmitir información y facilitar la comunicación- proporcionan al docente un eficaz soporte didáctico en el ámbito de la enseñanza presencial. Es evidente que la incorporación de las TIC ha acrecentado las diferencias ya existentes entre países y grupos sociales (Gros y Contreras, 2006). El papel que actualmente ocupan las TIC en la sociedad provocan que el profesor no sea el único dueño del conocimiento, sino un facilitador en la búsqueda del mismo (De la Torre, 2005). El uso de las nuevas tecnologías en el aula puede ayudar a que el profesorado sea más receptivo a los cambios en la metodología, así como incluya mejoras en la orientación y asesoramiento, en la organización y dinamización de grupos, en la motivación de los estudiantes, en el diseño y gestión de entornos de aprendizaje, en la creación de recursos, y en la introducción de una evaluación formativa. Entre las ventajas didácticas de las nuevas tecnologías en el aula está el fomento del trabajo cooperativo (Repáraz, Lara, Mir, y Orobiogoikoetxea, 2006), el trabajo personalizado, así como el trabajo con estudiantes de lugares alejados en el espacio. Si se emplean de este modo las TIC servirán para un cambio fundamental en la estructura de enseñanza (De la Torre, 2005).

Nos encontramos igualmente ante una necesaria formación del alumnado para el uso las TIC y de las plataformas de teleformación, la cual promoverá en los mismos una actitud crítica del uso de estos medios, logrando de esta forma no solo su manejo, sino también una calidad de la interacción tanto con los contenidos como con las otras personas que las utilizan en el transcurso de las prácticas educativas en las que se implica (Barberá y Badia, 2008).

El uso de estos recursos aumentan las opciones de experimentación en disciplinas como la geografía y la historia (Martín, 2011). Como opina Acosta (2010), la historia se encuentra en desventaja respecto a las ciencias experimentales, incluso con otras ciencias sociales, debido a la imposibilidad de reproducir los hechos históricos objetos de estudio. La introducción de las TIC da la oportunidad a los docentes de solventar

Evaluación del diseño e implementación de la metodología flipped-classroom en la formación del profesorado de ciencias sociales. José Monteagudo Fernández, Cosme Jesús Gómez Carrasco y Pedro Miralles Martínez.

Página 9 de 26 
algunos de estos problemas. Los principales argumentos esgrimidos para el uso de las nuevas tecnologías, y que pueden potenciar la enseñanza de las ciencias sociales, han girado en torno al aprendizaje autorregulado y significativo que permiten las TIC, la colaboración, tanto entre alumnos, como entre docentes y discentes, y el hecho de que la enseñanza pueda ser más particular e individualizada. También, como han mostrado Hernández Cardona (2011) o Pilar Rivero (2011), la utilización de imágenes virtuales, así como la interactividad que permiten las nuevas tecnologías o el uso de la multimedia expositiva, son uno de los principales elementos que potencian la enseñanza de la geografía y la historia. Es decir, es una opinión bastante generalizada que la utilización de las TIC y el camino hacia un aula virtual es un valioso recurso en el aprendizaje de las ciencias sociales en todos los niveles educativos (Osset, 2004).

La realidad aumentada y los códigos QR, junto a los MOOC's y otros entornos de aprendizaje están enriqueciendo los medios y recursos disponibles para el profesorado en la docencia universitaria. Entre las ventajas de la realidad aumentada, Cabero y Marín (2017) indican la capacidad de observación de un objeto desde diferentes puntos de vista, centrar la atención en los aspectos más significativos, o crear escenarios "artificiales" seguros. Además de estas características los anteriores autores insisten en la capacidad de ser utilizada en diferentes disciplinas, entre ellas las ciencias sociales, y en distintos niveles educativos (De Pedro Carracedo y Méndez, 2012; Bressler \& Bodzin, 2013; Prendes, 2015; Cabero \& Barroso, 2016).

La flipped-classroom (también conocida como clase inversa o aula invertida) es un método que consiste en invertir los métodos tradicionales de enseñanza, donde la instrucción propiamente dicha se da fuera del aula y los deberes dentro de clase (Bergmann \& Sams, 2012). Como indica Gil y Chiva (2016), también se le conoce como aula intercambiada (Wenier, 2012) o inversión de la clase. El cambio propuesto por estos trabajos es que esa inversión de la clase pueda generar en el alumnado la posibilidad de que se estructure su tiempo de aprendizaje de forma autónoma, ya que recibe el contenido de las sesiones teóricas en formato audiovisual y con algunos materiales complementarios con antelación. El alumnado debe organizarse para visualizar esos materiales previos al trabajo en clase, pudiendo reproducirlos tantas veces como desee. Como apuntan Casanova y Serrano (2017), el formato del vídeo explicativo tiene una gran potencialidad didáctica para el alumnado, ya que ellos tienen una gran motivación con todas las herramientas audiovisuales. Para el trabajo en clase se recomienda utilizar técnicas cooperativas que permitan la discusión de esos materiales entre el alumnado, y de éstos con el docente. Bergman y Sams (2012) defienden que este método permite mejorar la interacción entre docentes y estudiantes, ya que el docente adquiere un papel de guía en el trabajo realizado en el aula, tanto en el procesamiento de la información como en las actividades prácticas, simulaciones, debates etc. Esta interacción permite al docente responder a las necesidades emocionales y de aprendizaje del alumnado con mayor información en tiempo real, de manera que puede favorecer la retroalimentación en el proceso de asimilación del conocimiento.

A partir de 2012, el número de publicaciones científicas sobre flippled classroom ha aumentado año a año. En un estado de la cuestión reciente, se ha calculado que el $72 \%$

Evaluación del diseño e implementación de la metodología flipped-classroom en la formación del profesorado de ciencias sociales. José Monteagudo Fernández, Cosme Jesús Gómez Carrasco y Pedro Miralles Martínez.

Página 10 de 26 
de las experiencias e investigaciones publicadas tuvieron lugar en los Estados Unidos. En cambio, en países europeos hay una ausencia notable de estudios académicos (Uzunboylu \& Karagözlü, 2017). Cubrir esta laguna es otra de las metas del presente trabajo.

En cuanto a los métodos empleados, sobresalen los estudios experimentales y de caso. Se prefiere recopilar datos mediante pruebas, cuestionarios, exámenes, etc. El número de estudios cualitativos es alto, empleando el análisis de documentos, las entrevistas y otras herramientas alternativas de recopilación de datos cualitativos (Uzunboylu \& Karagözlü, 2017).

El planteamiento de varias flipped-classroom en la mencionada asignatura de "Metodología didáctica para la enseñanza de las ciencias sociales" ha permitido al alumnado tomar contacto con estas nuevas metodologías activas de aprendizaje a través de una experiencia directa. La metodología de la clase al revés o flipped-classroom forma parte de un movimiento general de la educación actual en el que el foco de atención del aula se centra en el alumnado y no en el profesor: el aula gira en torno a los estudiantes y la educación se personaliza hacia ellos. Para ello será fundamental la creación de materiales que permita personalizar el aprendizaje del alumnado; primar los procedimientos sobre los conceptos; y facilitar el acceso a la teoría.

Los pilares básicos que hay que tener en cuenta a la hora de planificar una intervención a través de la flippled-classroom según Gil y Chiva (2016) son los siguientes:

1. Ambiente flexible. Cada alumno trabaja a su propio ritmo. Esto permite atender mejor la diversidad en las aulas.

2. Cultura de aprendizaje. Los estudiantes se convierten en responsables de su aprendizaje.

3. Contenido intencionado. Aquí reside la causa del éxito o fracaso de esta metodología. Todo el proceso previo a la implementación de esta metodología se debe realizar de forma muy cuidadosa.

4. Educadores como guías. Tener la posibilidad de proporcionar retroalimentación inmediata a los estudiantes, asesorarles en su trabajo, y personalizar el proceso de enseñanza-aprendizaje.

En España hay varios autores que son referentes en la aplicación de esta metodología en el aula de ciencias sociales, como Antonio Lucero o Rosa Liarte. En sus respectivas páginas web se pueden explorar los procedimientos utilizados, sus vídeos y los feedback que han conseguido con el alumnado. Recientemente también se han publicado algunas experiencias en ciencias sociales como las de Benítez (2017) en Bachillerato, Casanova y Serrano (2017) en la formación del profesorado, o la investigación realizada por Vélez y González (2017) sobre la recepción de esta metodología en diferentes cursos de la ESO, Bachillerato y Grado de Educación Primaria. Una pauta común que muestran todas las propuestas y experiencias citadas es la buena valoración que hace el alumnado de este método, el incremento de su motivación en todo el proceso, y los resultados positivos en el aprendizaje de unos contenidos que con los métodos tradiciones suelen ser percibidos como tediosos y memorísticos por parte de los estudiantes.

Evaluación del diseño e implementación de la metodología flipped-classroom en la formación del profesorado de ciencias sociales. José Monteagudo Fernández, Cosme Jesús Gómez Carrasco y Pedro Miralles Martínez.

Página 11 de 26 


\section{Metodología}

\subsection{Objetivos de la investigación}

El objetivo principal de este trabajo es evaluar el diseño e implementación de varias flipped-classroom en la formación del profesorado de ciencias sociales en el Grado de Educación Primaria. Para ello se formularon una serie de objetivos específicos:

1. Conocer la experiencia que tenía el alumnado sobre esta metodología.

2. Analizar la valoración que realizan los estudiantes de los vídeos y materiales complementarios aportados a distancia por el profesorado.

3. Indagar, en el transcurso de la sesión presencial, la implicación del alumnado y la valoración de las actividades prácticas realizadas.

4. Averiguar la opinión final de los discentes sobre la flipped-classroom como método educativo.

\subsection{Contexto y participantes}

La propuesta de innovación y la investigación sobre los resultados obtenidos se ha realizado en la asignatura "Metodología didáctica para la enseñanza de las ciencias sociales" (código 1153 del Grado de Educación Primaria de la Facultad de Educación de la Universidad de Murcia). En una asignatura como ésta es imprescindible que el alumnado aprenda y experimente con nuevas metodologías de aprendizaje basadas en la actividad del alumnado, la construcción del conocimiento integrando diversas habilidades y conocimientos, y un papel del docente capaz de combinar diferentes estrategias y técnicas, así como gestionar diferentes ritmos de aprendizaje. Sería un error que el alumnado aprenda nuevas metodologías de enseñanza a través de métodos tradicionales. Por ello el objetivo principal del equipo docente es que el alumnado aprenda estas nuevas metodologías experimentando con ellas en su proceso de enseñanza-aprendizaje. Aunque esta metodología se implementó en los siete grupos donde se imparte docencia, para la investigación sobre los resultados obtenidos solamente contamos con los tres grupos, dos de mañana (grupo 1 y 3 ) y uno de tarde (grupo 7), en los que los tres profesores implicados en el proyecto, sin experiencia previa en la aplicación de esta estrategia metodológica, pasaron el cuestionario al alumnado, tratándose, por tanto, de un muestreo incidental. El número de participantes fue de 92, de un total de 174.

\subsection{Procedimiento didáctico y metodológico}

Para el diseño del planteamiento metodológico se seleccionaron cuatro temáticas clave, una por cada tema de la asignatura, sobre las que implementar las cuatro flippedclassroom. En ese diseño entraron en juego la búsqueda y selección de materiales ya elaborados en la Red; la creación de materiales audiovisuales propios; la planificación de la acción docente; y la recogida de pruebas que evidenciaran el resultado de estas experiencias.

Se crearon dos vídeos sobre la materia que se debía abordar en dos de las flippedclassroom (tema 3 y 4). Además se buscaron y seleccionaron materiales diversos (vídeos de YouTube, artículos periodísticos, artículos académicos) que pudieran complementar e introducir al alumnado en la materia que se iba a desarrollar en el aula a través del trabajo cooperativo en las cuatro flipped-classroom. Las temáticas de la

Evaluación del diseño e implementación de la metodología flipped-classroom en la formación del profesorado de ciencias sociales. José Monteagudo Fernández, Cosme Jesús Gómez Carrasco y Pedro Miralles Martínez.

Página 12 de 26 
flipped-classroom que se llevaron a cabo y sobre las que se buscaron los materiales fueron:

Tema 1. Metodología. Estrategias expositivas Vs Estrategias de indagación.

Tema 2. Recursos. Las fuentes primarias como recurso en las clases de ciencias sociales.

Tema 3. Las TIC. Los videojuegos.

Tema 4. La Unidad Didáctica. La evaluación.

Explicación del aula invertida:

http://one.elpais.com/playlist/nuevas-formulas-la-educacion-del-futuro/

Entrevista a Francisco Mora para introducir la neuroeducación:

https://www.youtube.com/watch?v=amUX26JLGS4

Entrevista a Roger Shrank para introducir las metodologías activas de aprendizaje:

https://www.youtube.com/watch?v=B4j6ZyQQE6o

Artículo periodístico sobre la relación entre emociones y conocimiento:

http://blogs.elpais.com/ayuda-al-estudiante/2013/12/la-neuroeducaciondemuestra-que-emocion-y-conocimiento-van-juntos.html

Artículo periodístico con una entrevista a Roger Shrank sobre metodologías activas de aprendizaje:

https://elpais.com/economia/2016/07/26/actualidad/1469530199_692638.ht $\mathrm{ml}$

Artículo periodístico sobre la necesidad de introducir el medio cercano en los procesos de aprendizaje:

http://blogs.elpais.com/ayuda-al-estudiante/2013/12/los-ni\%C3\%B1osdeben-empezar-a-aprenden-en-la-naturaleza-no-en-el-aula.html

Vídeo con la lectio del Dr. Joaquim Prats en su investidura como doctor Honoris Causa. Combates por la historia en educación.

https://www.youtube.com/watch?v=nHLH6OqIxdg

Tabla 1. Materiales complementarios utilizados. Elaboración propia.

Para la implementación de las flipped-classroom se realizaron varias reuniones entre el grupo docente de la asignatura (dos presenciales, y el resto virtuales) para planificar el desarrollo de las sesiones, los materiales que se mandarían a distancia al alumnado para su visualización, así como las actividades que se desarrollarían en el aula. En esas actividades principalmente se primó el debate dirigido sobre las principales temáticas

Evaluación del diseño e implementación de la metodología flipped-classroom en la formación del profesorado de ciencias sociales. José Monteagudo Fernández, Cosme Jesús Gómez Carrasco y Pedro Miralles Martínez.

Página 13 de 26 
abordadas en los vídeos y materiales, y la realización de ejercicios y prácticas en grupo, fomentando el trabajo cooperativo.

Los vídeos de creación propia y los materiales complementarios se enviaron con una semana de antelación al alumnado para que tuvieran tiempo suficiente para su visualización y lectura. Las cuatro flipped-classroom que se implementaron comenzaron con una debate dirigido en torno a las temáticas elegidas (métodos tradiciones Vs métodos de indagación; las fuentes primarias; los videojuegos; la evaluación). Después se realizaron ejercicios específicos para reforzar los contenidos.

Posteriormente se elaboró por parte del grupo docente una rúbrica en la que se evaluó tanto la participación en los debates dirigidos como los ejercicios que el alumnado debía entregar. Los resultados conseguidos en los debates fueron sensiblemente inferiores a los ejercicios entregados. Pese a que se animó a la discusión y el debate, la participación no fue muy elevada. Para conseguir los objetivos de investigación propuestos, relacionados con la evaluación de los resultados desde el punto de vista del alumnado, se elaboró un cuestionario con preguntas abiertas para ser contestado por los estudiantes al finalizar las cuatro flipped-classroom. Las preguntas fueron las siguientes:

1. ¿Conocías este método antes de tratarlo en la asignatura "Metodología didáctica para la enseñanza de las Ciencias Sociales? Si la respuesta es afirmativa indica a través de qué medio.

2. ¿Te han resultado útiles e interesantes los materiales (textos y vídeos) empleados? ¿Por qué?

3. ¿Cómo valoras el desarrollo de las sesiones presenciales y las actividades prácticas realizadas?

4. ¿Cómo evalúas la participación del alumnado?

5. En general, ¿cuál es tu valoración de la flipped-classroom como metodología?

A pesar de tratarse de un cuestionario de preguntas abiertas, la naturaleza de las propias cuestiones, así como las respuestas obtenidas, permiten una categorización dicotómica de estas últimas. Así se logra reducir el dato textual a un tratamiento y análisis de datos numéricos, donde interesa más la frecuencia que el propio contenido de las categorías, aunque no se descuida esto último.

Los datos en forma de respuestas libres a preguntas abiertas pueden ser procesados mediante el tratamiento cuantitativo de lo cualitativo, incluso a un nivel más complejo del que se presenta en estas líneas (Benzécri, 1973).

\section{Resultados}

La primera pregunta del cuestionario pasado a los estudiantes indagaba sobre si éstos tenían conocimiento previo del método flipped-classroom antes de su empleo en la asignatura "Metodología didáctica para la enseñanza de las ciencias sociales", en el curso 3. ${ }^{\circ}$ del Grado en Educación Primaria, y, si la respuesta era afirmativa, a través de qué medio habían obtenido dicho conocimiento previo. El gráfico 1 muestra que para el $81 \%$ del alumnado participante (75 sujetos) se estaba delante de un método de enseñanza desconocido, mientras que el 19\% restante (17 discentes) sí lo había abordado previamente en otras asignaturas de la carrera.

Evaluación del diseño e implementación de la metodología flipped-classroom en la formación del profesorado de ciencias sociales. José Monteagudo Fernández, Cosme Jesús Gómez Carrasco y Pedro Miralles Martínez.

Página 14 de 26 


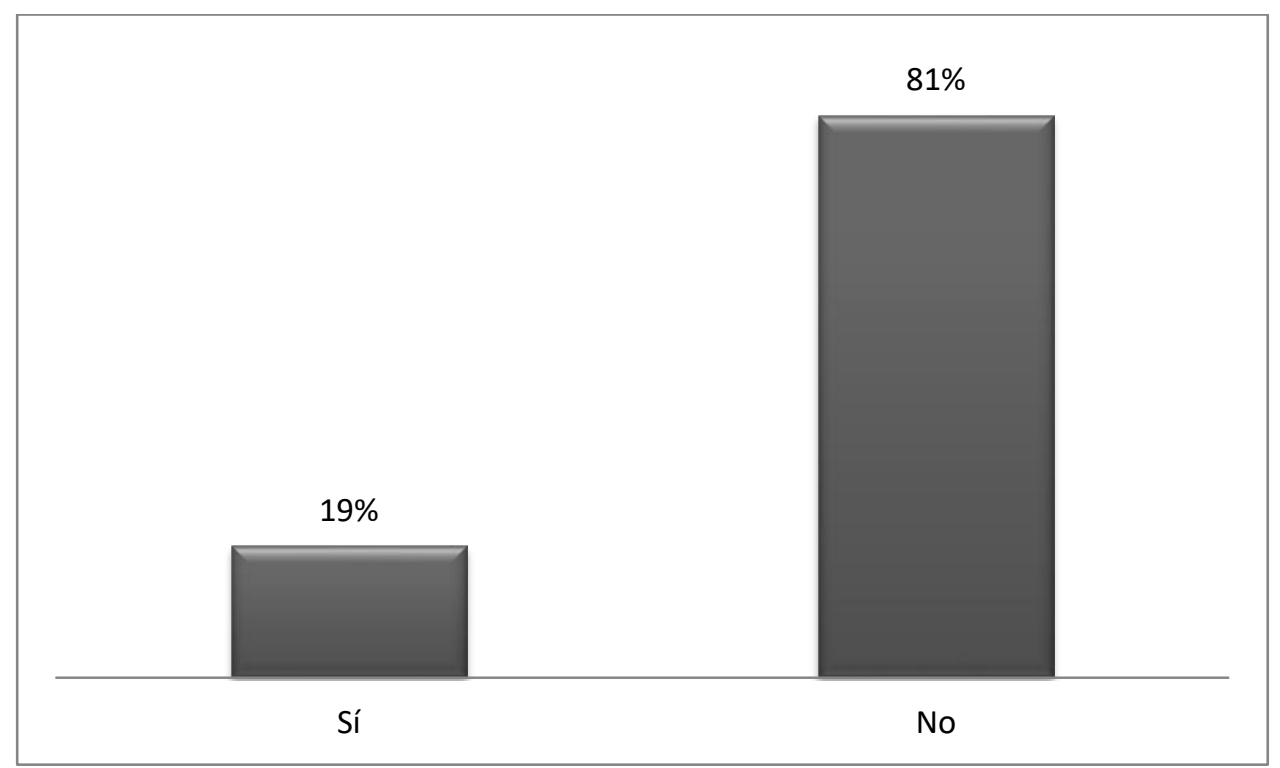

Gráfico 1. Porcentaje de alumnado que tenía conocimientos previos sobre flippedclassroom. Elaboración propia.

La segunda cuestión versaba sobre si los materiales empleados para desarrollar las sesiones propias de la flipped-classroom fuera del aula les habían parecido útiles e interesantes. Los resultados mostrados en el gráfico 2 no dejan lugar a dudas. Para el $100 \%$ del alumnado participante los materiales resultaron interesantes en la medida en que, según los aportes cualitativos de sus respuestas, ayudaban a captar la atención de los estudiantes, aumentaban la motivación y permitían un cambio en las sesiones presenciales tendente a mejorar la dinámica de clase, pudiendo así obtener mejores resultados finales en opinión del alumnado.

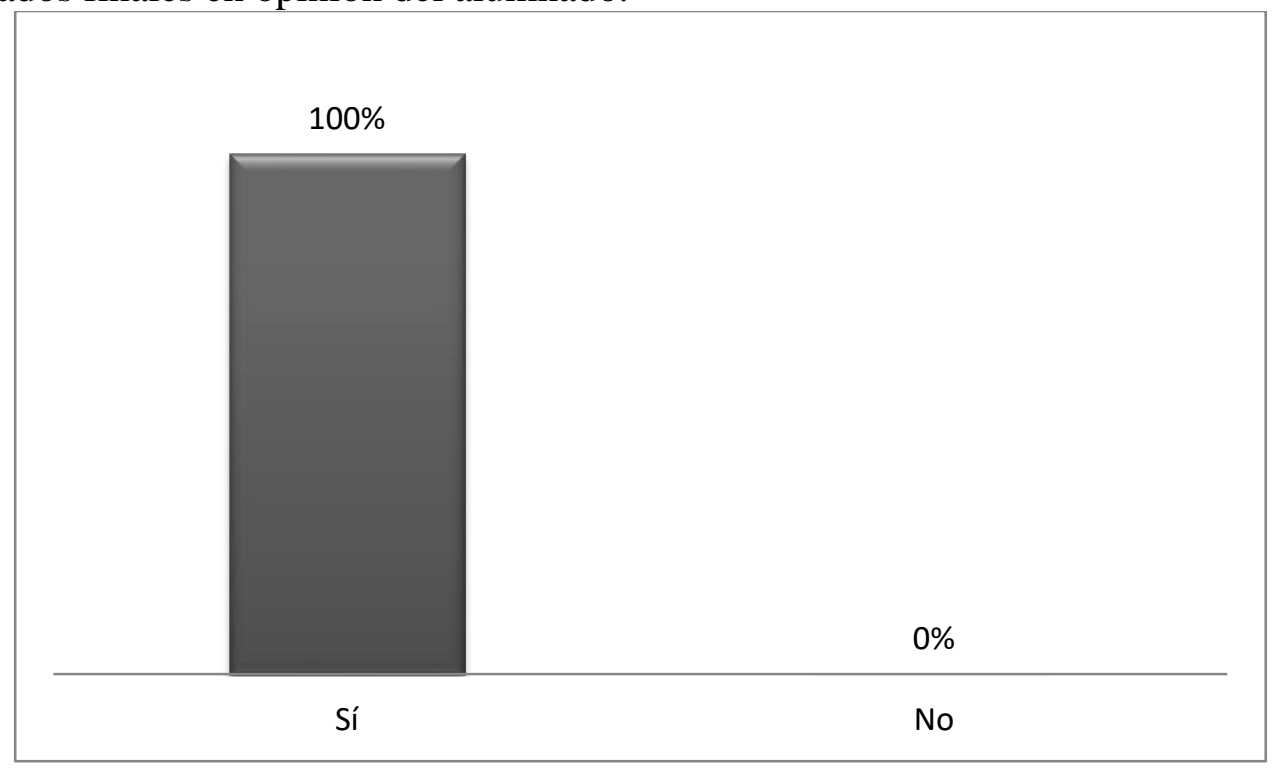

Gráfico 2. Porcentaje de alumnado a quien han resultado útiles e interesantes los materiales empleados. Elaboración propia.

Evaluación del diseño e implementación de la metodología flipped-classroom en la formación del profesorado de ciencias sociales. José Monteagudo Fernández, Cosme Jesús Gómez Carrasco y Pedro Miralles Martínez.

Página 15 de 26 
En consonancia con lo anterior, la valoración de las sesiones presenciales y las actividades prácticas propuestas por los docentes, tercera cuestión que se planteó al alumnado, arroja unos resultados positivos del 90\% (83 participantes), como puede apreciarse en el gráfico 3. Para el 10\% restante (9 discentes) las sesiones presenciales no se desarrollaron en todo su potencial debido a la escasa participación de los propios estudiantes, cuestión que precisamente se recogía en la pregunta número cuatro del cuestionario. En este sentido (Gráfico 4) comprobamos que el 88\% del alumnado consideró que había participado muy poco en las clases prácticas, empleando diversa terminología para referirse a esa escasa participación (baja, pasiva, mejorable), lo que a su juicio suponía un entorpecimiento en el desarrollo de las sesiones presenciales, pues en los temas a desarrollar y debatir los únicos que intervenían en la mayoría de ocasiones eran los propios profesores. Una parte significativa reconocía que los discentes no habían cumplido suficientemente con su trabajo fuera del aula para poder llevar a cabo las sesiones prácticas. Otros lo justificaban achacándolo a la vergüenza y miedo a la equivocación que sienten todavía a la hora de expresarse verbalmente en un aula delante de compañeros y docentes.

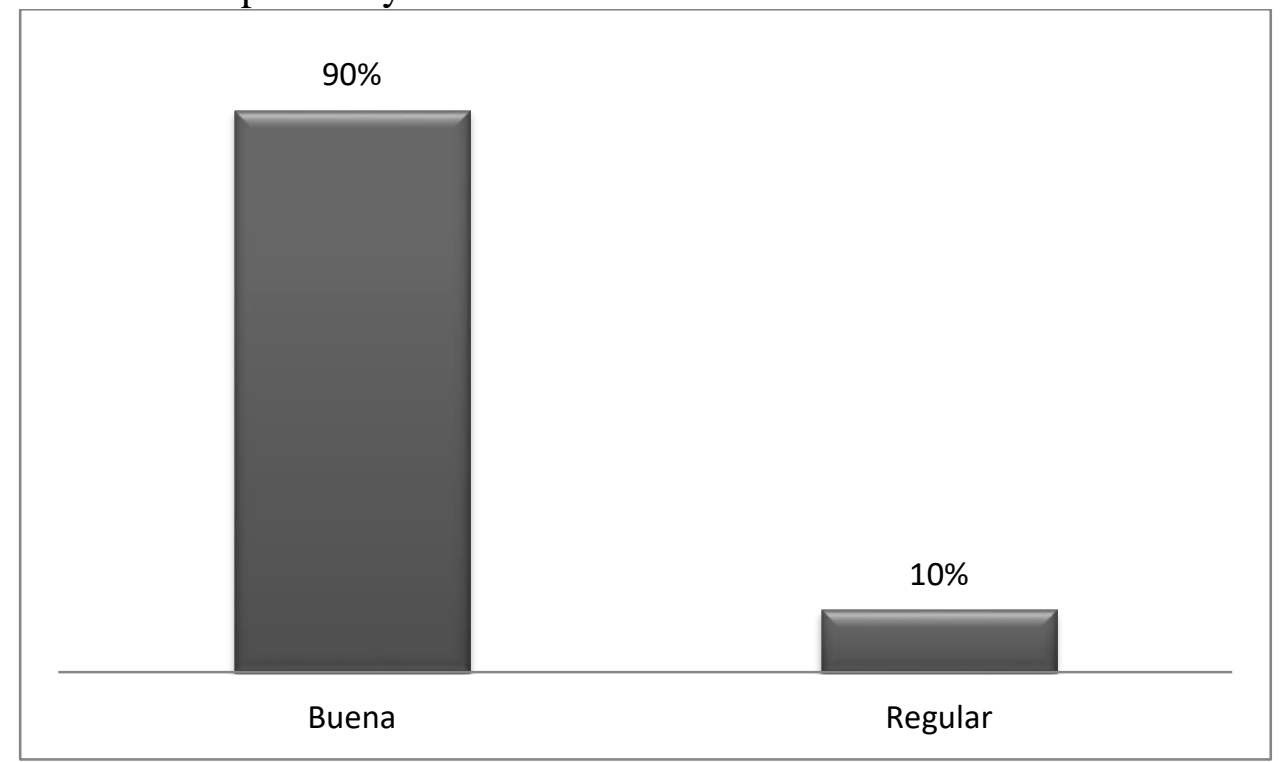

Gráfico 3. Valoración del desarrollo de las sesiones presenciales y las actividades prácticas realizadas. Elaboración propia.

Evaluación del diseño e implementación de la metodología flipped-classroom en la formación del profesorado de ciencias sociales. José Monteagudo Fernández, Cosme Jesús Gómez Carrasco y Pedro Miralles Martínez.

Página 16 de 26 


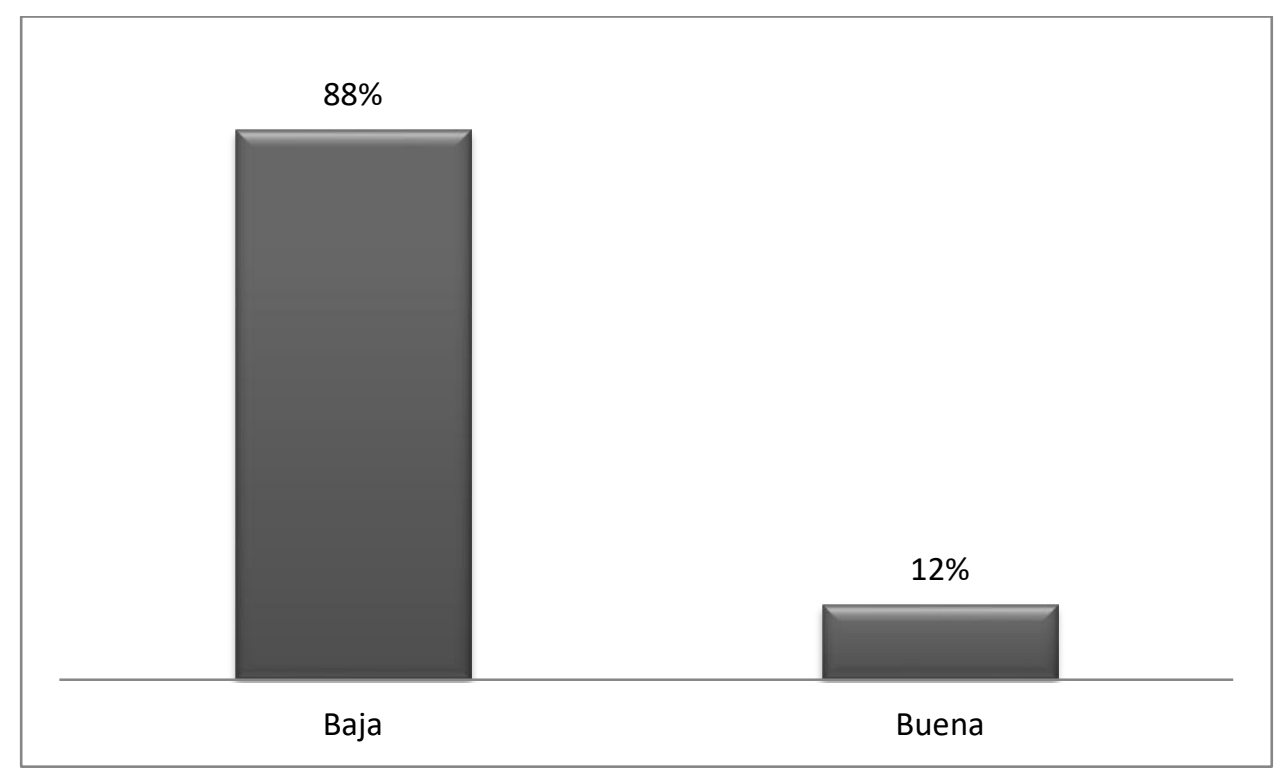

Gráfico 4. Valoración de la participación del alumnado. Elaboración propia.

Nos encontramos con lo que podría parecer una contradicción en las respuestas del alumnado cuando valoran positivamente las sesiones prácticas a la vez que critican su falta de participación en las mismas, algo que podría suponer un lastre para el propio desarrollo de dichas sesiones. Desde nuestra perspectiva entendemos que lo que ocurre es que el alumnado tiene una buena percepción de la preparación y desenvolvimiento de las sesiones prácticas por los profesores aunque es consciente de que su falta de implicación impide que se lleven a cabo con la profundidad que podría alcanzar.

Finalmente la última cuestión propuesta a los estudiantes fue que valoraran la flippedclassroom como metodología a implementar en el aula para conseguir aprendizajes significativos. Nuevamente un 100\% de los participantes (Gráfico 5) tenía un buen concepto de ella. Entre las justificaciones más frecuentes para una valoración positiva se encontraba el hecho de que a través de las clases invertidas el alumnado consideraba que se fomenta la autonomía, la participación y la actividad de los aprendices, adaptándose a los ritmos y diversidad de las aulas, lo que la hace ser más efectiva que otros métodos. Sin embargo, fruto de su experiencia, como estudiantes que son, también remarcaron la necesidad de que el docente emplee los recursos más adecuados para su puesta en práctica. Lo contrario podría acarrear problemas para lograr la implicación de los discentes.

Evaluación del diseño e implementación de la metodología flipped-classroom en la formación del profesorado de ciencias sociales. José Monteagudo Fernández, Cosme Jesús Gómez Carrasco y Pedro Miralles Martínez.

Página 17 de 26 


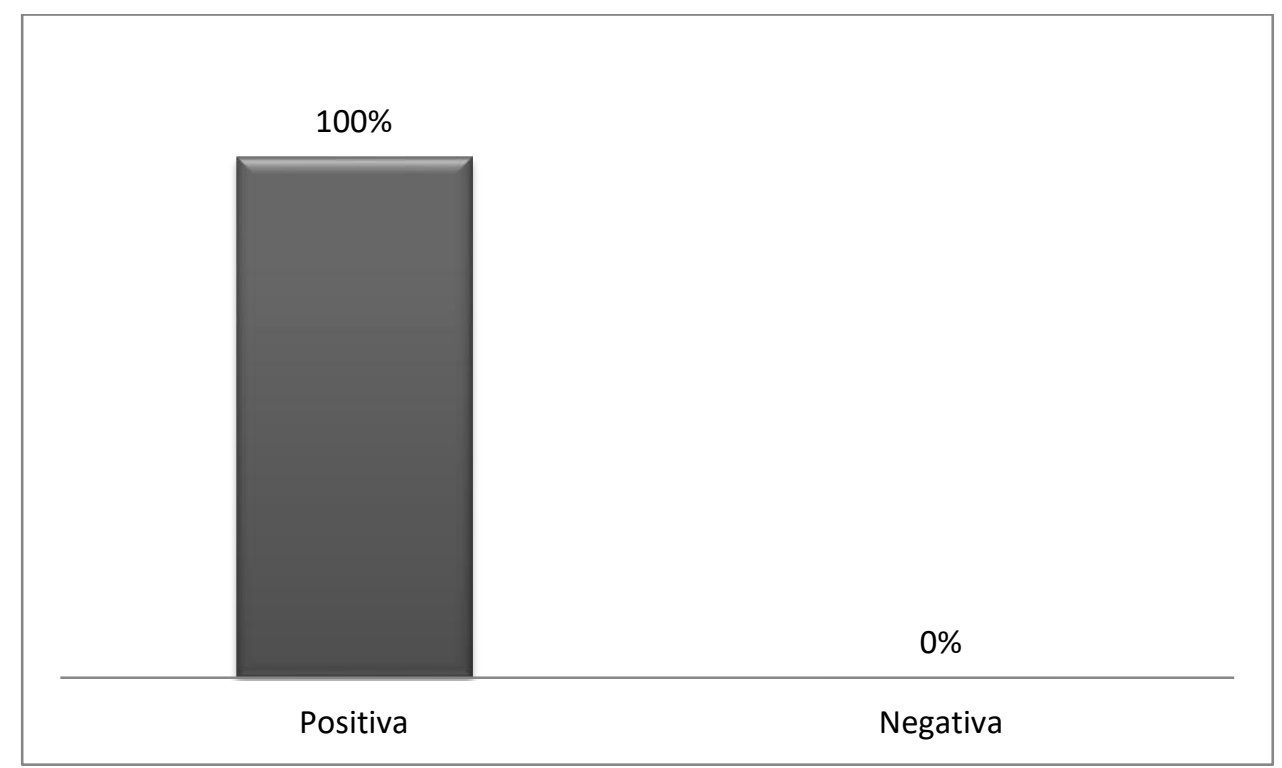

Gráfico 5. Valoración de la flipped-classroom como metodología. Elaboración propia.

\section{Discusión y conclusiones}

Una vez expuestos los resultados del estudio, pasamos a enunciar las conclusiones obtenidas, las cuales nos han ayudado a alcanzar los objetivos propuestos:

1. La gran mayoría del alumnado carecía de experiencia con el método de la flippedclassroom y lo desconocía como estrategia de enseñanza. De ahí la necesidad de seguir dando a conocer, trabajar y fomentar su uso entre los maestros en formación de educación primaria.

2. Los vídeos y materiales complementarios aportados a distancia por el profesorado para llevar a cabo las clases invertidas fueron valorados positivamente por todo el alumnado implicado, lo que demuestra el acierto del equipo docente en la selección de dichos materiales.

3. El transcurso de las sesiones presenciales fue bien valorado por el alumnado, sobre todo las actividades prácticas realizadas, no así la implicación de los propios estudiantes, quienes dejaron patente su falta de participación durante las sesiones.

4. El alumnado tenía una opinión positiva de la flipped-classroom como método educativo útil para el proceso de enseñanza-aprendizaje, al que otorga virtudes como el fomento de la autonomía en los estudiantes y el papel activo de estos, parecer resaltado en la última pregunta del cuestionario.

La experiencia desarrollada en las sesiones de la asignatura de Metodología para la enseñanza de las ciencias sociales, junto con los resultados que aporta el análisis del cuestionario pasado al alumnado, coincide con otras propuestas y experiencias llevadas a cabo con antelación para el caso de las ciencias sociales en otros niveles de enseñanza en lo que a la buena valoración que hace el alumnado de este método y el incremento de la motivación concierne (Benítez, 2017; Casanova y Serrano (2017); Vélez y González, 2017).

Evaluación del diseño e implementación de la metodología flipped-classroom en la formación del profesorado de ciencias sociales. José Monteagudo Fernández, Cosme Jesús Gómez Carrasco y Pedro Miralles Martínez.

Página 18 de 26 
Sin embargo la necesidad de seguir mejorando la práctica docente, de continuar dando a conocer la flipped-classroom como método que arroja buenos resultados y la reconocida escasa participación del alumnado, nos empuja a profundizar en la implementación de este método en cursos sucesivos. En este sentido, y teniendo en cuenta esta experiencia, se ha de incentivar la motivación y cooperación de los estudiantes a fin de que ellos lo experimenten y comprueben todo el potencial de las clases invertidas como medio para lograr aprendizajes significativos en todos los niveles de enseñanza.

La intención es la de hacer ver a una mayoría creciente de futuros y futuras docentes de educación primaria que disponen de una estrategia de enseñanza que, bien empleada, facilita y potencia los procesos de adquisición de conocimientos dentro del aula, incrementando el grado de compromiso e implicación de los estudiantes con el contenido del curso y mejorando su aprendizaje.

Presentación del artículo: 30 de agosto de 2017 Fecha de aprobación: 12 de diciembre de 2017 Fecha de publicación: 22 de diciembre de 2017

Monteagudo Fernández, J.; Gómez Carrasco, C. J., y Miralles Martínez P. (2017). Evaluación del diseño e implementación de la metodología flipped-classroom en la formación del profesorado de ciencias sociales. RED. Revista de Educación a Distancia, 55. Consultado el (dd/mm/aaaa) en http://www.um.es/ead/red/55/monteagudo_et_al.pdf

\section{FINANCIACIÓN}

El presente artículo es fruto de los proyectos de investigación "La evaluación de las competencias y el desarrollo de capacidades cognitivas sobre historia en Educación Secundaria Obligatoria" (EDU2015-65621-C3-2-R), financiado por el Ministerio de Economía y Competitividad; e "Implantación y evaluación de la metodología flipped-classroom en la asignatura "Metodología didáctica para la enseñanza de las ciencias sociales" del $3 .^{\circ}$ curso del Grado de Educación Primaria", al amparo de la convocatoria de la Unidad de Innovación de la Universidad de Murcia para promover proyectos y acciones de innovación y mejora en la Universidad de Murcia para el curso 2016/2017.

\section{FUNDING}

This paper is included within the research project titled "La evaluación de las competencias y el desarrollo de capacidades cognitivas sobre historia en Educación Secundaria Obligatoria" (EDU2015-65621-C3-2-R, financed by Ministerio de Economía y Competitividad, and "Implantación y evaluación de la metodología flipped-classroom en la asignatura "Metodología

Evaluación del diseño e implementación de la metodología flipped-classroom en la formación del profesorado de ciencias sociales. José Monteagudo Fernández, Cosme Jesús Gómez Carrasco y Pedro Miralles Martínez.

Página 19 de 26 
didáctica para la enseñanza de las ciencias sociales" del 3. curso del Grado de Educación Primaria", recognized by Unidad de Innovación de la Universidad de Murcia.

\section{Referencias bibliográficas}

Acosta, L. M. (2010). La enseñanza-aprendizaje de la historia en bachillerato y las TIC: la introducción de la estrategia Webquest. Proyecto Clio, 36.

Albalate, D., Fagueda, X., y Perdiguero, J. (2011) Éxito académico, características personales y proceso de Bolonia. Revista d'innovació docent universitària, 3, 1125.

Álvarez, S., Cuéllar, C., López, B., Adrada, C., Anguiano, R., Bueno, A., Comas, I. y Gómez, S. (2011). Actitudes de los profesores ante la información de las TIC en la práctica docente. Estudio de un grupo de la Universidad de Valladolid. Edutec, 35. Recuperado de (FECHA) http://edutec.rediris.es/revelec2/revelec35/.

Artal, J., Casanova, O, Serrano, R, y Romero, E. (2017). Dispositivos móviles y flippedclassroom. Una experiencia multidisciplinar de formación del profesorado. Edutec. Revista Electrónica de Tecnología Educativa, 59. Recuperado el 18/09/2017 de http://www.edutec.es/revista.

Banas, J. (2010). Teachers' Attitudes toward Technology. Considerations. Community \& Junior College Libraries, 16(2), 114-127.

Barberá, E. y Badia, A. (2008). Perspectivas actuales sobre la calidad educativa de los procesos de E-A que incorporan las TIC. En T. Barberá (Ed.). Cómo valorar la calidad de la enseñanza basada en las TIC (pp. 29-45). Barcelona: Graó.

Benítez, M. (2017). Flipped-classroom para las mentes del mañana en el aula de Geografía e Historia. En R. Martínez, R. García-Moris, y C. R. García (Eds.). Investigación en didáctica de las ciencias sociales. Retos, preguntas y líneas de investigación (pp. 349-356). Córdoba: Universidad de Córdoba.

Benzécri, J.P. (1973). L’Analyse des Dones. París: Dunod.

Bergmann, J. \& Sams, A. (2012). Flip your classroom. Reach every student in every class every day. Washington: ISTE.

Biggs, J. (2005). Calidad del aprendizaje universitario. Madrid: Narcea.

Biggs, J., Kember, D. \& Leung, D. Y. P. (2001). The revised two-factor Study Process Questionnaire: R-SPQ-2F. British Journal of Educational Psychology. 71, 133149.

Evaluación del diseño e implementación de la metodología flipped-classroom en la formación del profesorado de ciencias sociales. José Monteagudo Fernández, Cosme Jesús Gómez Carrasco y Pedro Miralles Martínez.

Página 20 de 26 
Bolívar, J. M. y Rojas F. (2008). Los estilos de aprendizaje y el locus de control en estudiantes que inician estudios superiores y su vinculación con el rendimiento académico. Investigación y Postgrado, 23(3), 199-215.

Boyd, C. P. (2000). Historia patria: política, historia e identidad nacional en España, 1875-1975. Barcelona: Pomares-Corredor.

Bressler, D. M., \& Bodzin, A. M. (2013). A mixed methods assessment of students' flow experiences during a mobile augmented reality science game. Journal of Computer Assisted Learning, 29(6), 505-517. doi: 10.1111/jcal.12008

Cabero, J. (2014). Formación del profesorado universitario en TIC. Aplicación del método Delphi para la selección de los contenidos formativos. Educación XX1, 17(1), 1-132. doi: http://doi.org/10.5944/educxx1.17.1.10707

Cabero, J. y Marín, V. (2014). Miradas sobre la formación del profesorado en TIC. Enl@ce Revista venezolana de Información, Tecnología y Conocimiento, 11(2), 11-24.

Cabero, J. y Marín, V. (2017). Dispositivos móviles y realidad aumentada en el aprendizaje del alumnado universitario. RIED. Revista Iberoamericana de Educación a Distancia, 20(2), 167-185. doi: http://dx.doi.org/10.5944/ried.20.2.17245

Cabero, J., \& Barroso, J. (2016). The educational possibilities of Augmented Reality. NAER. New Approaches in Educational Research, 5(1), 44-50. doi: https://doi.org/10.7821/naer.2016.1.140

Cano, E. (2005). Cómo mejorar las competencias de los docentes. Guía para la autoevaluación y el desarrollo de las competencias del profesorado. Barcelona: Graó.

Casanova, O. y Serrano, R. (2017). Flipped-classroom en ciencias sociales. Íber. Didáctica de las Ciencias Sociales, Geografía e Historia, 88, 47-52.

Curry, M.J., \& Lillis, T.M. (2005). Issues in academic writing in Higher Education. En C. Coffin, M.J. Curry, S. Goodman, A. Hewings, T.M. Lillis, \& J. Swann, Teaching Academic Writing (pp. 1-18). London/New York: Routledge.

De La Torre, J. L. (2005). Las nuevas tecnologías en las clases de ciencias sociales del siglo XXI. Quaderns Digitals, 37. http://www.quadernsdigitals.net/

De Pedro Carracedo, J., y Méndez, C. L. M. (2012). Realidad Aumentada: Una Alternativa Metodológica en la Educación Primaria Nicaragüense. IEEE-RITA, 7, 102-108. Recuperado de http://www.redusoi.org/docs/ LibroActasCAFVIR2011.pdf\#page $=300$

Evaluación del diseño e implementación de la metodología flipped-classroom en la formación del profesorado de ciencias sociales. José Monteagudo Fernández, Cosme Jesús Gómez Carrasco y Pedro Miralles Martínez.

Página 21 de 26 
Dean, V., De Caro, A. y Lagomarsino, M. (2013). El modelo T-Pack como buenas prácticas para integrar las TIC en la educación a distancia. Trabajo Final del Curso ProTic2013. Universidad de Morón.

Del Moral (2012). Conocimiento didáctico general para el diseño y desarrollo de experiencias de aprendizaje significativas en la formación del profesorado. Profesorado. Revista de curriculum y formación del profesorado 16 (2), 469500.

Del Moral, E. y Villalustre, L. (2010). La formación del profesor 2.0. Desarrollo de competencias tecnológicas para la Escuela 2.0. Revista Miscelánea de Investigación, 23, 59-70.

Demirbas, O. \& Demirkan, H. (2007) Learning styles of design students and the relationship of academic performance and gender in design education. Learning and Instruction, 17, 345-359.

Entwistle, N. J. (2000). Approaches to studying and levels of understanding: The influences of teaching and assessment. En J. C. Smart y W. G. Tierney (Eds.), Higher education: Handbook of theory and research (pp. 156-218). New York: Springer.

Entwistle, N. \& McCune, V. (2004). The conceptual bases of Study strategy inventories. Educational Psychology Review, 16(4), 325-345. Recuperado de (19/10/2017) http://www.springerlink.com/content/127747602358j724

Fielden, J. (2001). Higher education staff development continuing mission. En Thematic dabte of the follow-up to the World Conference on High Education. UNESCO.

Fuentes, L. (2003). Análisis de la relación entre estilos de pensamiento y estrategias de comprensión lectora en una muestra de estudiantes universitarios. Revista de Investigación-Educación, 18, 29-43.

García, M. (2016). ¿Cómo aprenden los alumnos en su primer año de universidad al inicio y al final del curso? REDU - Revista de Docencia Universitaria, 14(1), $27-49$.

García, T., Arias, O, Rodríguez, C., Fidalgo, R., y Robledo, P. (2017). Metodologías activas y desarrollo de competencias en estudiantes universitarios con diferentes estilos de pensamiento. RIDU. Revista d'Innovació Docent Universitària, 9, 6680.

Gargallo, B., Garfella P. y Pérez, E. (2006). Enfoques de aprendizaje y rendimiento académico en estudiantes universitarios, Bordón, 58 (3), 47-61.

Gil, J. y Chiva, Ó. (2016). Flipped-classroom (clase invertida). En Ó. Chiva y M. Martí (Coords). Métodos pedagógicos activos y globalizadores. Barcelona: Graó.

Evaluación del diseño e implementación de la metodología flipped-classroom en la formación del profesorado de ciencias sociales. José Monteagudo Fernández, Cosme Jesús Gómez Carrasco y Pedro Miralles Martínez.

Página 22 de 26 
González, J. L.; del Rincón, B.; del Rincón, D. A. (2011). Estructura latente y Consistencia interna del R-SPQ-2F: Reinterpretando los enfoques de aprendizaje en el EEES. Revista de Investigación Educativa, 29 (2), 277-293.

Gros, B. y Contreras, D. (2006). La alfabetización digital y el desarrollo de competencias. Revista Iberoamericana de Educación, 42, 103-125.

Guzmán, F.; García, E. (2017). La alfabetización académica de los futuros maestros. Un estudio comparativo en varias universidades españolas. Revista de Investigación Educativa, 35(2), 317-335. doi: http://dx.doi.org/10.6018/rie.35.2.246011

Hernández Cardona, F. X. (2011). La iconografía en la didáctica de las ciencias sociales. Íber. Didáctica de las ciencias sociales, geografía e historia, 68, 7-16.

Hernández Pina, F. (1999). Los enfoques de aprendizaje en el contexto de la evaluación de la calidad de las universidades (Informe final). Programa sectorial de promoción general del conocimiento. Murcia: Universidad de Murcia.

Hernández Pina, F. (2002). Docencia e investigación en educación superior. Revista de Investigación Educativa, 20(2), 270-301.

Hernández Pina, F., Hervás, R. M., Maquilón, J. J., García Sanz, M. P. y Martínez, P. (2002). Consistencia entre motivos y estrategias de aprendizaje en estudiantes universitarios. Revista de Investigación Educativa, 20 (2), 487-510.

Hernández Pina, F., Rosário, P., Cuesta, J. D., Martínez, P. y Ruiz, E. (2006). Promoción del aprendizaje estratégico y competencias de aprendizaje en estudiantes de primero de universidad: evaluación de una intervención. Revista de Investigación Educativa, 24 (2), 615-632.

Jimoyiannis, A. \& Komis, V. (2007). Examining teachers' beliefs about ICT in education. Implications of a teacher preparation programme. Teacher Development, 11(2), 149-173.

Juárez, C. S., Rodríguez, G., Escoto, Ma C. y Luna, E. (2017). Relación de estilos y estrategias de aprendizaje con el rendimiento académico en estudiantes universitarios. Journal of Learning Styles, 9 (17), 268-288.

Kember, D. (2000). Misconceptions about the learning approaches, motivation and study practices of Asian students. Higher Education, 40, 99-121.

León, M ${ }^{\mathrm{a}}$ J. y Crisol, E. (2011). Diseño de cuestionarios (oppumaugr y opeumaugr): la opinión y la percepción del profesorado y los estudiantes sobre el uso de las metodologías activas en la universidad. Profesorado. Revista de currículum y formación del profesorado, 15(2), 271-298.

Evaluación del diseño e implementación de la metodología flipped-classroom en la formación del profesorado de ciencias sociales. José Monteagudo Fernández, Cosme Jesús Gómez Carrasco y Pedro Miralles Martínez.

Página 23 de 26 
López, M. C. (2007). El espacio europeo de educación superior y su impacto en la evaluación del proceso enseñanza-aprendizaje. Educaçao Temática Digital, 9, 50- 67.

Magadán, C. (2012). Clase 4: El desafío de integrar actividades, proyectos y tareas con TIC. En VV.AA. Enseñar y aprender con TIC, Especialización docente de nivel superior en educación y TIC (pp. 1-14). Buenos Aires: Ministerio.

Margalef, L. y Pareja, N. (2008). Un camino sin retorno: estrategias metodológicas de aprendizaje activo. Revista Interuniversitaria de Formación del Profesorado, 22(3), 47-62.

Martín, C. (2011). Tecnologías digitales interactivas y didáctica de las ciencias, Íber. Didáctica de las Ciencias Sociales, geografía e historia, 68, 33-39.

Mishra, P. \& Koehler, M. J. (2006). Technological Pedagogical Content Knowledge: A Framework for Teacher Knowledge. Teachers College Record, 108(6), 10171054.

Muñoz-Repiso, A. G. V., Gómez-Pablos, V. B., González, M. C., Martín, S. C., Rodero, L. M. G., Martín, A. H. y Marcos, J. J. M. (2015). La formación del profesorado universitario en Tecnologías de la Información y la Comunicación en la Universidad de Salamanca. RELATEC: Revista Latinoamericana de Tecnología Educativa, 14(1), 75-88.

Ojeda, M. L., Carreras, O., Vázquez, C. M. y Mate, A. (2010). Elaboración de los materiales didácticos necesarios para la adaptación de la enseñanza de hematología al Espacio Europeo de Educación Superior. Revista de Investigación Educativa, 28 (2), 313-324.

Osset, J. (2004). Las nuevas tecnologías en ciencias sociales. Un viaje hacia el aula virtual. Íber. Didáctica de las Ciencias Sociales, geografía e historia, 41, 57-67.

Pérez-Garzón, S. (2008). ¿Por qué enseñamos geografía e historia? ¿Es tarea educativa la construcción de identidades? Historia de la educación, 27, 37-55.

Perrenoud, P. (2004). Diez nuevas competencias para enseñar. Barcelona: Graó.

Perrenoud, P. (2008). Construir las competencias, ¿es darle la espalda a los saberes? REDU - Revista de Docencia Universitaria, número monográfico sobre Formación centrada en competencias II. 6(2), 1-16.

Prats, J. y Santacana, J. (2011). ¿Por qué y para qué enseñar historia? En G. Galicia (Cood.), Enseñanza y aprendizaje de la historia en Educación Básica (pp. 1868). México: Secretaría de Educación Pública.

Evaluación del diseño e implementación de la metodología flipped-classroom en la formación del profesorado de ciencias sociales. José Monteagudo Fernández, Cosme Jesús Gómez Carrasco y Pedro Miralles Martínez.

Página 24 de 26 
Prendes, C. (2015). Realidad aumentada y educación: análisis de experiencias prácticas. Pixel-Bit. Revista de Medios y Educación, 46, 187-203. doi: http://dx.doi.org/10.12795/pixelbit.2015.i46.12

Repáraz, Ch., Lara, S., Mir, J. I. y Orobiogoikoetxea, E. (2006). Empleo de WebQuest para la elaboración de vídeos científicos en geografía (4 ${ }^{\circ}$ de la ESO). La indagación científica y el aprendizaje cooperativo. Íber. Didáctica de las ciencias sociales, geografía e historia, 49, 109-122.

Rivero, P. (2011). Un estudio sobre la efectividad de la multimedia expositiva para el aprendizaje de la Historia. Enseñanza de las ciencias sociales. Revista de investigación, 10, 45-50.

Rodríguez-Correa, M. y González-Sanmamed, M. (2013). La gestión del cambio institucional en las Universidades a través de las TIC. Revista de Docencia Universitaria, 11(3), 363-384.

Romero, A.; Hidalgo, Ma D.; González, F.; Carrillo, E.; Pedraja, Mª García, J.; Pérez, M. (2013). Enfoques de aprendizaje en estudiantes universitarios: comparación de resultados con los cuestionarios ASSIST y R-SPQ-2F. Revista de Investigación Educativa, 31(2), 375-391.

Rué, J. (2008) Formar en competencias en la universidad: entre la relevancia y la banalidad. REDU - Revista de Docencia Universitaria, Núm. Monográfico sobre Formación centrada en competencias, 6(1), 1-19.

Ruiz I.; Rubia, B.; Anguita, R. y Fernández, E. (2010). Formar al profesorado inicialmente en habilidades y competencias en TIC: perfiles de una experiencia colaborativa. Revista Educación, 352, 149-178.

Sánchez, Ma P. (Coord.). (2010). Técnicas docentes y sistemas de evaluación en Educación Superior. Madrid: Narcea.

Santacana, J. (2005). Reflexiones en torno al laboratorio escolar en ciencias sociales. Íber. Didáctica de las Ciencias sociales, Geografía e Historia, 43, 7-14.

Santisteban, A. y Pagès, J. (Coords.). (2011). Didáctica del Conocimiento del Medio Social y Cultural en la Educación Primaria. Ciencias Sociales para comprender, pensar y actuar. Madrid: Síntesis.

Selwyn, N., Marriott, N. \& Marriott, P. (2002). Home Computers \& University ICT Use. Journal of Computer Assisted Learning, 1(18), 44-45.

Torra, I.; de Corral, I.; Pérez, M.J.; Triadó, X.; Pagès, T.; Valderrama, E.; Màrquez, M.D.; Sabaté, S.; Solà, P.; Hernàndez, C.; Sangrà, A.; Guàrdia, G.; Estebanell, M.; Patiño, J.; González, A.; Fandos, M.; Ruiz, N.; Iglesias, M.C. y Tena, A. (2012). Identificación de competencias docentes que orienten el desarrollo de

Evaluación del diseño e implementación de la metodología flipped-classroom en la formación del profesorado de ciencias sociales. José Monteagudo Fernández, Cosme Jesús Gómez Carrasco y Pedro Miralles Martínez.

Página 25 de 26 
planes de formación dirigidos a profesorado universitario. REDU - Revista de Docencia Universitaria, Número monográfico dedicado a Competencias docentes en la Educación Superior. 10 (2), 21-56. Recuperado el 9 de mayo de $2013 \mathrm{http}: / /$ redaberta.usc.es/redu

Vélez, F. J. y González, C. (2017). Flipped-classroom en las aulas de ciencias sociales. En R. Martínez, R. García-Moris, y C. R. García (Eds.). Investigación en didáctica de las ciencias sociales. Retos, preguntas y líneas de investigación (pp. 810-816). Córdoba: Universidad de Córdoba.

Wenier, L. (2012). The future of our schools. Chicago: Haymarkets Books.

Yániz, C. (2008). Las competencias en el currículo universitario: implicaciones para diseñar el aprendizaje y para la formación del profesorado. REDU - Revista de Docencia Universitaria. Núm. Monográfico sobre Formación centrada en competencias. 6(1), 1-13.

Zabalza, M.Á. (2002). La enseñanza universitaria. El escenario y sus protagonistas. Madrid: Narcea.

Zahonero, A. y Martín, M. (2012). Formación integral del profesorado: hacia el desarrollo de competencias personales y de valores en los docentes. Tendencias pedagógicas, 20, 51-70.

Evaluación del diseño e implementación de la metodología flipped-classroom en la formación del profesorado de ciencias sociales. José Monteagudo Fernández, Cosme Jesús Gómez Carrasco y Pedro Miralles Martínez.

Página 26 de 26 